\title{
LARGE TIME BEHAVIOR OF SOLUTIONS TO THE ISENTROPIC COMPRESSIBLE FLUID MODELS OF KORTEWEG TYPE IN $\mathbb{R}^{3 *}$
}

\author{
ZHONG TAN $^{\dagger}$ AND YONG WANG ${ }^{\ddagger}$
}

\begin{abstract}
We consider the long-time behavior and optimal decay rates of global strong solutions to the isentropic compressible Navier-Stokes-Korteweg system in $\mathbb{R}^{3}$. When the regular initial data belong to the Sobolev space $H^{l+1}\left(\mathbb{R}^{3}\right) \cap \dot{B}_{1, \infty}^{-s}\left(\mathbb{R}^{3}\right) \times H^{l}\left(\mathbb{R}^{3}\right) \cap \dot{B}_{1, \infty}^{-s}\left(\mathbb{R}^{3}\right)$ with $l \geq 3$ and $s \in[0,1]$, we show that the density and momentum of the system converges to its equilibrium state at the rates $(1+t)^{-\frac{3}{4}-\frac{s}{2}}$ in the $L^{2}$-norm or $(1+t)^{-\frac{3}{2}-\frac{s}{2}}$ in the $L^{\infty}$-norm, respectively, which are proved to be optimal for the compressible Navier-Stokes-Korteweg system.
\end{abstract}

Key words. Korteweg system, compressible Navier-Stokes equations, optimal decay rates, energy estimates.

MSC. 35Q35, 35L65, 76N10, 76N15, 76D45.

\section{Introduction}

The compressible Navier-Stokes-Korteweg system governs the motions of the compressible isothermal viscous capillary fluids. We study its initial value problem (IVP):

$$
\left\{\begin{array}{l}
\partial_{t} \rho+\nabla \cdot m=0, \\
\partial_{t} m+\nabla \cdot\left(\frac{m \otimes m}{\rho}\right)+\nabla P(\rho)-\mu \Delta\left(\frac{m}{\rho}\right)-\nu \nabla \operatorname{div}\left(\frac{m}{\rho}\right)=\kappa \rho \nabla \Delta \rho, \\
\left.(\rho, m)\right|_{t=0}=\left(\rho_{0}, m_{0}\right),
\end{array}\right.
$$

for $(x, t) \in \mathbb{R}^{3} \times \mathbb{R}^{+}$. The unknown variables $\rho, m$ represent the density and momentum of the fluid, respectively, and $P=P(\rho)$ is the pressure satisfying $P^{\prime}(\rho)>0$ for $\rho>0$. The constants $\mu>0, \nu \geq 0$ are the viscosity coefficients and $\kappa>0$ is the capillary coefficient.

Since Korteweg [15] first introduced the theory of capillarity with diffuse interfaces which was derived rigorously by Dunn and Serrin [5], many mathematicians have contributed to the development of Navier-Stokes-Korteweg system. Meantime, many important results about the existence and uniqueness of (weak, strong or smooth) solutions can be found in $[1,2,7,8,9,16]$ and the references therein. We refer to $[1,7]$ for the global existence of weak solutions, [16] for the local existence of strong solutions, $[8,9]$ for the local and global existences of classical solutions, and [2] for the global existence of smooth solutions in the critical Besov space.

There have been many important advancements in the investigation of large time behavior of global solutions to the compressible Navier-Stokes system. For instance, Matsumura and Nishida [19] obtained the optimal $L^{2}$ convergence rate for the compressible viscous and heat-conductive fluid in $\mathbb{R}^{3}$ without external force:

$$
\|(\rho-\bar{\rho}, u, \theta-\bar{\theta})(t)\|_{L^{2}} \leq C_{0}(1+t)^{-\frac{3}{4}},
$$

${ }^{*}$ Received: November 7, 2011; accepted (in revised form): March 8, 2012. Communicated by Song Jiang.

Supported by National Natural Science Foundation of China- NSAF (Grant No. 10976026).

${ }_{\dagger}^{\dagger}$ School of Mathematical Sciences Xiamen University, China (ztan85@163.com).

${ }^{\ddagger}$ Corresponding author: School of Mathematical Sciences Xiamen University, China (wangyongxmu@163.com). 
if the small initial perturbations belongs to $H^{3} \cap L^{1}$, and Ponce [21] proved the optimal $L^{p}$ convergence rate

$$
\left\|\nabla^{k}(\rho-\bar{\rho}, u, \theta-\bar{\theta})(t)\right\|_{L^{p}} \leq C_{0}(1+t)^{-\frac{3}{2}\left(1-\frac{1}{p}\right)-\frac{k}{2}},
$$

for $2 \leq p \leq \infty$ and $0 \leq k \leq 2$, if the small initial perturbations belong to $H^{l} \cap W^{l, 1}$ with $l$ a sufficiently large integer. Li-Zhang [18] considered the Cauchy problem for the compressible Navier-Stokes equations when the regular initial data belong to $H^{l}\left(\mathbb{R}^{3}\right) \cap$ $\dot{B}_{1, \infty}^{-s}\left(\mathbb{R}^{3}\right)$, with $l \geq 4$ and $s \in[0,1]$, and showed the optimal time decay rates

$$
\|(\rho-\bar{\rho}, m)(t)\|_{L^{2}} \leq C_{0}(1+t)^{-\frac{3}{4}-\frac{s}{2}} .
$$

Zeng [28] showed the convergence of global solution to the nonlinear Burgers' diffusive wave. Wang and Tan [26] proved the optimal $L^{2}$ and $L^{p}(p \geq 2)$ decay rates for the classical solutions and their spatial derivatives for the compressible Navier-StokesKorteweg system. Zhang, $\mathrm{Li}$, and Zhu [27] considered the compressible non-isentropic Navier-Stokes-Poisson system and analyzed the influence of an internal electric field on the qualitative behaviors of solutions. Li, Matsumura, and Zhang [17] proved the global existence and optimal decay rates of the solutions to the compressible Navier-Stokes-Poisson equations. Recently, Guo-Wang [6] and Wang [24] developed a general energy method for proving the optimal time decay rates of the solutions to the dissipative equations in the whole space by introducing the negative Sobolev space. Finally, concerning the long time decay rates of global solutions for the half space and exterior domain or for the general external force, we refer to the papers $[10,11,12,13,14,16,18,22,25]$.

In this paper, we conduct a linearized analysis by decomposing the velocity field as the potential and rotational parts, compared with the known results in $[17,18,22$, $25,26,27]$. This technique makes the linearized analysis simpler, which is more helpful for the non-isentropic model as in [23]. At the same time, the nonlinear analysis in Section 3 becomes clearer, since we divide the equations of motion of (3.1) into a simplified equation and a well-known heat equation by this technique.

Before we state the main results, let us first recall the definition of $\dot{B}_{1, \infty}^{-s}$.

Let $\psi: \mathbb{R}^{3} \rightarrow[0,1]$ be a radial smooth cut-off function valued in $[0,1]$ such that

$$
\psi(\xi)= \begin{cases}1, & |\xi| \leq \frac{3}{4}, \\ \text { smooth, }, \frac{3}{4}<|\xi|<\frac{4}{3}, \\ 0, & |\xi| \geq \frac{4}{3} .\end{cases}
$$

Let $\varphi(\xi)$ be the function

$$
\varphi(\xi):=\psi\left(\frac{\xi}{2}\right)-\psi(\xi)
$$

Thus, $\psi$ is supported in the ball $\left\{\xi \in \mathbb{R}^{3}:|\xi| \leq \frac{4}{3}\right\}$, and $\varphi$ is also a smooth cut-off function valued in $[0,1]$ and supported in the shell $\left\{\xi \in \mathbb{R}^{3}: \frac{3}{4} \leq|\xi| \leq \frac{8}{3}\right\}$. By construction, we have

$$
\sum_{k \in \mathbb{Z}} \varphi\left(2^{-k} \xi\right)=1, \quad \text { if } \xi \neq 0
$$

Definition 1.1. We denote by $\dot{B}_{1, \infty}^{-s}$ the space of distributions which is the completion of $\mathcal{S}\left(\mathbb{R}^{3}\right)$ by the following norm:

$$
\|f\|_{\dot{B}_{1, \infty}^{-s}}:=\sup _{k \in \mathbb{Z}} 2^{-s k}\left\|\dot{\Delta}_{k} f\right\|_{L^{1}}
$$


where

$$
\dot{\Delta}_{k} f:=\varphi\left(2^{-k} D\right) f=2^{3 k} \int_{\mathbb{R}^{3}}\left(\mathscr{F}^{-1} \varphi\right)\left(2^{k} y\right) f(x-y) d y,
$$

and $\mathscr{F}(f)$ or $\hat{f}$ denotes the Fourier transform of the function $f$.

For the global existence and large time behavior of strong solutions, we have the following:

Theorem 1.2. Let $P^{\prime}(\rho)>0$ for $\rho>0$ and denote $n:=\rho-\bar{\rho}$. Assume that $n_{0}=$ $\rho_{0}-\bar{\rho} \in H^{l+1}\left(\mathbb{R}^{3}\right) \cap \dot{B}_{1, \infty}^{-s}\left(\mathbb{R}^{3}\right), m_{0} \in H^{l}\left(\mathbb{R}^{3}\right) \cap \dot{B}_{1, \infty}^{-s}\left(\mathbb{R}^{3}\right), l \geq 3$, and $s \in[0,1]$, with $\delta:=$ $\left\|n_{0}\right\|_{H^{l+1} \cap \dot{B}_{1, \infty}^{-s}}+\left\|m_{0}\right\|_{H^{l} \cap \dot{B}_{1, \infty}^{-s}}$ small enough. Then there is a unique global solution $(\rho, m)$ to the IVP (1.1) satisfying

$$
\begin{gathered}
\rho-\bar{\rho} \in C^{0}\left(\mathbb{R}^{+}, H^{l+1}\left(\mathbb{R}^{3}\right)\right) \cap C^{1}\left(\mathbb{R}^{+}, H^{l}\left(\mathbb{R}^{3}\right)\right), \\
m \in C^{0}\left(\mathbb{R}^{+}, H^{l}\left(\mathbb{R}^{3}\right)\right) \cap C^{1}\left(\mathbb{R}^{+}, H^{l-2}\left(\mathbb{R}^{3}\right)\right),
\end{gathered}
$$

and

$$
\left\|\partial_{x}^{k}(\rho-\bar{\rho})(t)\right\|_{L^{2}\left(\mathbb{R}^{3}\right)}+\left\|\partial_{x}^{k} m(t)\right\|_{L^{2}\left(\mathbb{R}^{3}\right)} \leq C \delta(1+t)^{-\frac{3}{4}-\frac{s}{2}-\frac{|k|}{2}},
$$

for $|k|=0,1$, where $C$ is a positive constant independent of time.

It should be noted that the time decay rates above are optimal. Indeed, we shall establish the lower bound decay rate for the global solution.

TheOREM 1.3. Under the assumption of Theorem 1.2, then, the global solution $(\rho, m)$ given by Theorem 1.2 satisfies

$$
c_{1}(1+t)^{-\frac{3}{4}-\frac{s}{2}} \leq\|(\rho-\bar{\rho})(t)\|_{L^{2}\left(\mathbb{R}^{3}\right)}+\|m(t)\|_{L^{2}\left(\mathbb{R}^{3}\right)} \leq C(1+t)^{-\frac{3}{4}-\frac{s}{2}},
$$

where $c_{1}, C$ are positive constants independent of time.

With additional regularity given for the initial data, we can also prove the optimal $L^{p}$ time decay rate for the global classical solution.

Theorem 1.4. Let $P^{\prime}(\rho)>0$ for $\rho>0$. Assume that $\rho_{0}-\bar{\rho} \in H^{l+1}\left(\mathbb{R}^{3}\right) \cap \dot{B}_{1, \infty}^{-s}\left(\mathbb{R}^{3}\right)$, $m_{0} \in H^{l}\left(\mathbb{R}^{3}\right) \cap \dot{B}_{1, \infty}^{-s}\left(\mathbb{R}^{3}\right)$, and $s \in[0,1]$, with $\delta:=\left\|\rho_{0}-\bar{\rho}\right\|_{H^{l+1} \cap \dot{B}_{1, \infty}^{-s}}+\left\|m_{0}\right\|_{H^{l} \cap \dot{B}_{1, \infty}^{-s}}$ small enough. Then there is a unique global classical solution $(\rho, m)$ to the IVP (1.1) satisfying

$$
\begin{gathered}
\|(\rho-\bar{\rho})(t)\|_{L^{p}\left(\mathbb{R}^{3}\right)} \leq C \delta(1+t)^{-\frac{3}{2}\left(1-\frac{1}{p}\right)-\frac{s}{2}}, \quad \text { if } l \geq 4, \\
\|m(t)\|_{L^{p}\left(\mathbb{R}^{3}\right)} \leq C \delta(1+t)^{-\frac{3}{2}\left(1-\frac{1}{p}\right)-\frac{s}{2}}, \quad \text { if } l \geq 5,
\end{gathered}
$$

for $p \in[2,+\infty]$.

REMark 1.5. Since the initial data belong to the homogeneous Besov space $\dot{B}_{1, \infty}^{-s}\left(\mathbb{R}^{3}\right)$ with $s \in[0,1]$, and we get the inequality

$$
\sup _{\xi \in \mathbb{R}^{3} \backslash\{0\}}\left(|\xi|^{-s}\left|\hat{n}_{0}(\xi)\right|+|\xi|^{-s}\left|\hat{m}_{0}(\xi)\right|\right) \leq C\left\|\left(n_{0}, m_{0}\right)\right\|_{\dot{B}_{1, \infty}^{-s}\left(\mathbb{R}^{3}\right)},
$$


it is not difficult to find that the initial data, being in the Besov space, have better behavior in the low frequency (see Section 2). So we obtain the faster time decay rates of the global solution $(n, m)(x, t)$ as in Theorem 1.2.

REMARK 1.6. In the paper Li-Zhang [18], there is an assumption

$$
\left|\hat{n}_{0}(\xi)\right|>c_{0} \delta|\xi|^{s} \text { and } \hat{m}_{0}=0 \quad \text { for } 0 \leq|\xi| \ll 1, \quad c_{0}>0,
$$

which is necessary to gain the optimal time decay rates as in Theorem 1.3. However, because of the influence of the Korteweg term, we can obtain the optimal time decay rates by a straightforward computation in the present paper.

NOTATION 1.7. In the following parts of the paper, $C>0$ and $c_{i}>0$, with $i \geq 1$ an integer, denote the generic positive constants independent of time.

The present paper is structured as follows. Section 2 is devoted to establishing the $L^{2}$ time decay rate of the global solution for the linearized system. We prove Theorem 1.2 and Theorem 1.3 in Section 3. In Section 4, we show the $L^{p}$ time decay rates.

\section{The linearized system}

In this section, we consider the Cauchy problem for the linearized Navier-StokesKorteweg system:

$$
\left\{\begin{array}{l}
\partial_{t} n+\nabla \cdot m=0, \\
\partial_{t} m-\mu \Delta m-\nu \nabla \operatorname{div} m+\gamma \nabla n-\kappa \nabla \Delta n=0, \\
\left.(n, m)\right|_{t=0}:=\left(\rho_{0}-\bar{\rho}, m_{0}\right), \quad x \in \mathbb{R}^{3},
\end{array}\right.
$$

where $\gamma=P^{\prime}(\bar{\rho})$. Introducing the Leray projector $\mathcal{P}:=I d+\nabla(-\Delta)^{-1} \operatorname{div}$ on divergence-free vector fields, and $\mathcal{P}^{\perp}:=I d-\mathcal{P}$, the first two equations of the system (2.1) translate into

$$
\left\{\begin{array}{l}
\partial_{t} n+\nabla \cdot \mathcal{P}^{\perp} m=0 \\
\partial_{t} \mathcal{P}^{\perp} m-\mu \Delta \mathcal{P}^{\perp} m-\nu \nabla \operatorname{div} \mathcal{P}^{\perp} m+\gamma \nabla n-\kappa \nabla \Delta n=0, \\
\partial_{t} \mathcal{P} m-\mu \Delta \mathcal{P} m=0 .
\end{array}\right.
$$

Note that the equation for $\mathcal{P} m$ reduces to an ordinary equation, independent from the others. Moreover, if we denote by $\Lambda^{s}$ the pseudo differential operator defined by $\Lambda^{s} y:=\mathscr{F}^{-1}\left(|\xi|^{s} \hat{y}(\xi)\right)$, it is equivalent to study $\mathcal{P}^{\perp} m$ or $y:=\Lambda^{-1} \operatorname{div} m$ and $\mathcal{P} m$ or $\omega:=\Lambda^{-1} \operatorname{curl} m$ (with $\left.(\operatorname{curl} z)_{i}^{j}=\partial_{i} z^{j}-\partial_{j} z^{i}\right)$. So we are led to consider:

$$
\left\{\begin{array}{l}
\partial_{t} n+\Lambda y=0 \\
\partial_{t} y-(\mu+\nu) \Delta y-\gamma \Lambda n-\kappa \Lambda^{3} n=0 \\
\partial_{t} \omega-\mu \Delta \omega=0
\end{array}\right.
$$

Indeed, as the definitions of $y, \omega$, and the relation

$$
m=-\Lambda^{-1} \nabla y-\Lambda^{-1} \operatorname{div} \omega
$$

involve pseudo-differential operators of degree zero, the estimates in the space $H^{l}\left(\mathbb{R}^{3}\right)$ for the original function $m$ will be the same as for $(y, \omega)$.

This section is devoted to the proof of the following results. 
Proposition 2.1. Let $n_{0} \in H^{l+1}\left(\mathbb{R}^{3}\right) \cap \dot{B}_{1, \infty}^{-s}\left(\mathbb{R}^{3}\right), m_{0} \in H^{l}\left(\mathbb{R}^{3}\right) \cap \dot{B}_{1, \infty}^{-s}\left(\mathbb{R}^{3}\right), l \geq 3, s \in$ $[0,1]$, and $(n, m)$ satisfy the system (2.1). Let $y=\Lambda^{-1} \operatorname{div} m$ and $\omega=\Lambda^{-1}$ curl $m$. Then there exists a constant $C$ such that for $0 \leq|k| \leq l$,

$$
\begin{aligned}
& \left\|\partial_{x}^{k} \omega(t)\right\|_{L^{2}\left(\mathbb{R}^{3}\right)} \leq C(1+t)^{-\frac{3}{4}-\frac{s}{2}-\frac{|k|}{2}}\left(\left\|\left(n_{0}, m_{0}\right)\right\|_{\dot{B}_{1, \infty}^{-s}\left(\mathbb{R}^{3}\right)}\right. \\
& \left.+\left\|\left(\partial_{x}^{k} n_{0}, \partial_{x}^{k} m_{0}\right)\right\|_{L^{2}\left(\mathbb{R}^{3}\right)}\right), \\
& \|n(t)\|_{L^{2}\left(\mathbb{R}^{3}\right)} \leq C(1+t)^{-\frac{3}{4}-\frac{s}{2}}\left(\left\|\left(n_{0}, m_{0}\right)\right\|_{\dot{B}_{1, \infty}^{-s}\left(\mathbb{R}^{3}\right)}+\left\|\left(n_{0}, m_{0}\right)\right\|_{L^{2}\left(\mathbb{R}^{3}\right)}\right) \\
& \left\|\partial_{x}^{|k|+1} n(t)\right\|_{L^{2}\left(\mathbb{R}^{3}\right)} \leq C(1+t)^{-\frac{3}{4}-\frac{s}{2}-\frac{|k|+1}{2}}\left(\left\|\left(n_{0}, m_{0}\right)\right\|_{\dot{B}_{1, \infty}^{-s}\left(\mathbb{R}^{3}\right)}\right. \\
& \left.+\left\|\left(\partial_{x}^{|k|+1} n_{0}, \partial_{x}^{k} m_{0}\right)\right\|_{L^{2}\left(\mathbb{R}^{3}\right)}\right), \\
& \left\|\partial_{x}^{k} y(t)\right\|_{L^{2}\left(\mathbb{R}^{3}\right)}+\left\|\partial_{x}^{k} m(t)\right\|_{L^{2}\left(\mathbb{R}^{3}\right)} \\
& \leq C(1+t)^{-\frac{3}{4}-\frac{s}{2}-\frac{|k|}{2}}\left(\left\|\left(n_{0}, m_{0}\right)\right\|_{\dot{B}_{1, \infty}^{-s}\left(\mathbb{R}^{3}\right)}+\left\|\left(\partial_{x}^{|k|+1} n_{0}, \partial_{x}^{k} m_{0}\right)\right\|_{L^{2}\left(\mathbb{R}^{3}\right)}\right) \text {. }
\end{aligned}
$$

REMARK 2.2. Because the third equation of (2.3) is an independent heat equation, we easily obtain the estimate of $\omega$ by using the Fourier transform.

Proof. The estimate for $\omega$ is obvious, so let us focus on the first two equations of (2.3). Taking the Fourier transform with respect to the space variable yields

$$
\frac{d}{d t}\left(\begin{array}{c}
\hat{n} \\
\hat{y}
\end{array}\right)=A(\xi)\left(\begin{array}{c}
\hat{n} \\
\hat{y}
\end{array}\right), \text { with } A(\xi):=\left(\begin{array}{cc}
0 & -|\xi| \\
\kappa|\xi|^{3}+\gamma|\xi|-(\mu+\nu)|\xi|^{2}
\end{array}\right) .
$$

The characteristic polynomial of $A(\xi)$ is $\lambda^{2}+(\mu+\nu)|\xi|^{2} \lambda+\kappa|\xi|^{4}+\gamma|\xi|^{2}$, the eigenvalues of which are

$$
\lambda_{ \pm}(\xi)=-\frac{\eta}{2}|\xi|^{2} \pm \frac{1}{2} \sqrt{\left(\eta^{2}-4 \kappa\right)|\xi|^{4}-4 \gamma|\xi|^{2}}
$$

where $\eta=\mu+\nu$.

So,

$$
(\hat{n}, \hat{y})^{T}=e^{t A} \cdot\left(\hat{n_{0}}, \hat{y_{0}}\right)^{T} .
$$

The semigroup $e^{t A}$ is expressed as

$$
e^{t A}=e^{\lambda_{+} t} P_{+}+e^{\lambda_{-} t} P_{-},
$$

where the projection operators $P_{ \pm}$can be computed as

$$
P_{ \pm}=\frac{A(\xi)-\lambda_{\mp} I}{\lambda_{ \pm}-\lambda_{\mp}} .
$$

By a direct computation, we have

$$
\begin{aligned}
e^{t A}:= & \hat{G}_{1}(\xi, t)+\hat{G}_{2}(\xi, t) \\
= & {\left[\begin{array}{ll}
\frac{\lambda_{+} e^{\lambda_{-}}-\lambda_{-} e^{\lambda_{+} t}}{\lambda_{+}-\lambda_{-}} & \frac{e^{\lambda_{-} t}-e^{\lambda} t}{\lambda_{+}-\lambda_{-}}|\xi| \\
\frac{e^{\lambda_{+}}-e^{\lambda_{-}}}{\lambda_{+}-\lambda_{-}} \gamma|\xi| & \frac{\lambda_{+} e^{\lambda_{-}}-\lambda_{-} e^{\lambda_{+}}}{\lambda_{+}-\lambda_{-}}
\end{array}\right] } \\
& +\left[\begin{array}{cc}
0 & 0 \\
\frac{e^{\lambda_{+} t}-e^{\lambda_{-}}}{\lambda_{+}-\lambda_{-}} \kappa|\xi|^{3} & \frac{e^{\lambda_{-}-e^{\lambda_{+}}}}{\lambda_{+}-\lambda_{-}} \eta|\xi|^{2}
\end{array}\right] .
\end{aligned}
$$


The representation above holds for $|\xi| \neq 0, \frac{2 \gamma}{\sqrt{\eta^{2}-4 \kappa}}$ when $\kappa<\kappa^{*}$; and for $|\xi| \neq 0$ when $\kappa \geq \kappa^{*}$, where

$$
\kappa^{*}=\frac{\eta^{2}}{4} \equiv \frac{(\mu+\nu)^{2}}{4}
$$

We denote

$$
b=\frac{1}{2} \sqrt{4 \gamma|\xi|^{2}-\left(\eta^{2}-4 \kappa\right)|\xi|^{4}} .
$$

For $\kappa \geq \kappa^{*}, b>0$ is a real number for any fixed $|\xi| \neq 0$, hence

$$
\lambda_{ \pm}(\xi)=-\frac{\eta}{2}|\xi|^{2} \pm b i
$$

that is, the eigenvalues of the linear system in Fourier transform are not real. Therefore, in this case we have

$$
\begin{gathered}
\frac{e^{\lambda_{+} t}-e^{\lambda_{-} t}}{\lambda_{+}-\lambda_{-}}=\frac{\sin (b t)}{b} e^{-\frac{\eta}{2}|\xi|^{2} t} \\
\frac{\lambda_{+} e^{\lambda_{-} t}-\lambda_{-} e^{\lambda_{+} t}}{\lambda_{+}-\lambda_{-}}=\left[\cos (b t)+\frac{\eta}{2} \frac{\sin (b t)}{b}|\xi|^{2}\right] e^{-\frac{\eta}{2}|\xi|^{2} t} .
\end{gathered}
$$

Thus we can show the pointwise estimates for $e^{t A}$ explicitly. While for $0<\kappa<\kappa^{*}$, (2.10) and (2.11) still hold for $|\xi| \ll 1$ but do not hold globally. For the high frequencies $|\xi| \gg 1, b$ is a pure imaginary number, so that the eigenvalues are real.

Now we study the behavior of $e^{t A}$ for both the low frequency and high frequency. We simply denote by $\hat{g}_{11}, \hat{g}_{12}, \hat{g}_{21}, \hat{g}_{22}$ the four components of $\hat{G}_{1}$, and by $\hat{g}_{1}, \hat{g}_{2}$ the nonzero components of $\hat{G}_{2}$. We shall estimate them term by term and divide the arguments into two cases in terms of the value of the capillary coefficient $\kappa$. Let $R>0$ be any fixed constant.

Case 1: $\kappa \geq \kappa^{*}$. For $|\xi| \geq R$, using the fact that

$$
\left.\left.\left|\frac{\sin (b t)}{b}\right| \xi\right|^{2} e^{-\frac{\eta}{2}|\xi|^{2} t}|\leq t| \xi\right|^{2} e^{-\frac{\eta}{2}|\xi|^{2} t} \leq \frac{4}{\eta} e^{-\frac{\eta}{4}|\xi|^{2} t},
$$

and substituting (2.10), (2.11) into (2.8), we deduce that, setting $\eta^{\prime}=\frac{\eta}{4}$,

$$
\begin{aligned}
& \left|\hat{g}_{11}(\xi, t), \hat{g}_{22}(\xi, t), \hat{g}_{2}(\xi, t)\right| \leq C e^{-\eta^{\prime}|\xi|^{2} t}, \quad\left|\hat{g}_{12}(\xi, t), \hat{g}_{21}(\xi, t)\right| \leq C|\xi|^{-1} e^{-\eta^{\prime}|\xi|^{2} t}, \\
& \left|\hat{g}_{1}(\xi, t)\right| \leq C|\xi| e^{-\eta^{\prime}|\xi|^{2} t}, \quad|\xi| \geq R .
\end{aligned}
$$

While for $|\xi| \leq R$, noticing that $b=O(|\xi|)$, we easily obtain

$$
\begin{aligned}
& \left|\hat{g}_{11}(\xi, t), \hat{g}_{22}(\xi, t), \hat{g}_{12}(\xi, t), \hat{g}_{21}(\xi, t), \hat{g}_{2}(\xi, t)\right| \leq C e^{-\eta^{\prime}|\xi|^{2} t}, \\
& \left|\hat{g}_{1}(\xi, t)\right| \leq C|\xi|^{2} e^{-\eta^{\prime}|\xi|^{2} t}, \quad|\xi| \leq R .
\end{aligned}
$$

Case 2: $0<\kappa<\kappa^{*}$. Observe that if we define $a=\eta-\sqrt{\eta^{2}-4 \kappa}$, then $a>0$ and

$$
\operatorname{Re}\left(\lambda_{ \pm}(\xi)\right) \leq-\frac{a}{2}|\xi|^{2}, \quad \forall \xi \in \mathbb{R}^{3} .
$$


Now for $|\xi| \geq R$, since $\left|\lambda_{ \pm}\right|,\left|\lambda_{+}-\lambda_{-}\right|=O\left(|\xi|^{2}\right)$, we derive from the expression (2.8) and (2.15) that, setting $a^{\prime}=\frac{a}{2}$,

$\left|\hat{g}_{11}(\xi, t), \hat{g}_{22}(\xi, t), \hat{g}_{2}(\xi, t)\right| \leq C e^{-a^{\prime}|\xi|^{2} t}, \quad\left|\hat{g}_{12}(\xi, t), \hat{g}_{21}(\xi, t)\right| \leq C|\xi|^{-1} e^{-a^{\prime}|\xi|^{2} t}$, $\left|\hat{g_{1}}(\xi, t)\right| \leq C|\xi| e^{-a^{\prime}|\xi|^{2} t}, \quad|\xi| \geq R$.

While for $|\xi| \leq R$, since $\left|\lambda_{ \pm}\right|,\left|\lambda_{+}-\lambda_{-}\right|=O(|\xi|)$, we derive from the expression (2.8) and (2.15) that

$$
\begin{aligned}
& \left|\hat{g}_{11}(\xi, t), \hat{g}_{22}(\xi, t), \hat{g}_{12}(\xi, t), \hat{g}_{21}(\xi, t), \hat{g}_{2}(\xi, t)\right| \leq C e^{-a^{\prime}|\xi|^{2} t} \\
& \left|\hat{g}_{1}(\xi, t)\right| \leq C|\xi|^{2} e^{-a^{\prime}|\xi|^{2} t}, \quad|\xi| \leq R
\end{aligned}
$$

We may conclude from (2.13), (2.14), (2.16), and (2.17) that for any $\kappa>0$ there exists some $\beta:=\beta(\kappa)>0$ such that

$$
\left|\hat{g}_{11}(\xi, t), \hat{g}_{22}(\xi, t), \hat{g}_{12}(\xi, t), \hat{g}_{21}(\xi, t), \hat{g}_{1}(\xi, t), \hat{g}_{2}(\xi, t)\right| \leq C e^{-\beta|\xi|^{2} t},|\xi| \leq R
$$

and

$$
\begin{aligned}
& \left|\hat{g}_{11}(\xi, t), \hat{g}_{22}(\xi, t), \hat{g}_{2}(\xi, t)\right| \leq C e^{-\beta|\xi|^{2} t}, \quad\left|\hat{g}_{12}(\xi, t), \hat{g}_{21}(\xi, t)\right| \leq C|\xi|^{-1} e^{-\beta|\xi|^{2} t}, \\
& \left|\hat{g}_{1}(\xi, t)\right| \leq C|\xi| e^{-\beta|\xi|^{2} t}, \quad|\xi| \geq R .
\end{aligned}
$$

Now, by the expression (2.8) we have

$$
\hat{n}(\xi, t)=\hat{g}_{11} \hat{n}_{0}+\hat{g}_{12} \hat{y}_{0}, \quad \hat{y}(\xi, t)=\hat{g}_{21} \hat{n}_{0}+\left(\hat{g}_{22}+\hat{g}_{2}\right) \hat{y}_{0}+\hat{g}_{1} \hat{n}_{0} .
$$

Let $R>0$ be a fixed constant as before. By the pointwise estimates (2.18), (2.19), together with the Parseval theorem and Hausdorff-Young's inequality, we obtain

$$
\begin{aligned}
\|n(t)\|_{L^{2}\left(\mathbb{R}^{3}\right)}^{2}= & \int_{\mathbb{R}^{3}}\left|\hat{g}_{11}(\xi, t) \hat{n}_{0}(\xi)+\hat{g}_{12}(\xi, t) \hat{y}_{0}(\xi)\right|^{2} d \xi \\
\leq & C \int_{|\xi| \leq R} e^{-\beta|\xi|^{2} t}\left(\left|\hat{n}_{0}\right|^{2}+\left|\hat{y}_{0}\right|^{2}\right) d \xi \\
& \quad+C e^{-\beta R^{2} t} \int_{|\xi| \geq R}\left(\left|\hat{n}_{0}\right|^{2}+|\xi|^{-2}\left|\hat{y}_{0}\right|^{2}\right) d \xi \\
\leq & C\left\|\left(n_{0}, y_{0}\right)\right\|_{\dot{B}_{1, \infty}^{-s}\left(\mathbb{R}^{3}\right)}^{2} \int_{|\xi| \leq R} e^{-\beta|\xi|^{2} t}|\xi|^{2 s} d \xi \\
& \quad+C e^{-\beta R^{2} t}\left\|\left(n_{0}, y_{0}\right)\right\|_{L^{2}\left(\mathbb{R}^{3}\right)}^{2} \\
\leq & C(1+t)^{-\frac{3}{2}-s}\left\|\left(n_{0}, y_{0}\right)\right\|_{L^{2}\left(\mathbb{R}^{3}\right) \cap \dot{B}_{1, \infty}^{-s}\left(\mathbb{R}^{3}\right)}^{\leq} \\
\leq & C(1+t)^{-\frac{3}{2}-s}\left\|\left(n_{0}, m_{0}\right)\right\|_{L^{2}\left(\mathbb{R}^{3}\right) \cap \dot{B}_{1, \infty}^{-s}\left(\mathbb{R}^{3}\right)}^{2} \\
\left\|\partial_{x}^{|k|+1} n(t)\right\|_{L^{2}\left(\mathbb{R}^{3}\right)}^{2}= & \int_{\mathbb{R}^{3}}|\xi|^{2(|k|+1)}\left|\hat{g}_{11}(\xi, t) \hat{n}_{0}(\xi)+\hat{g}_{12}(\xi, t) \hat{y}_{0}(\xi)\right|^{2} d \xi \\
\leq & C \int_{|\xi| \leq R} e^{-\beta|\xi|^{2} t}|\xi|^{2(|k|+1)}\left(\left|\hat{n}_{0}(\xi)\right|^{2}+\left|\hat{y}_{0}(\xi)\right|^{2}\right) d \xi \\
& \quad+C \int_{|\xi| \geq R} e^{-\beta|\xi|^{2} t}\left(|\xi|^{2(|k|+1)}\left|\hat{n}_{0}(\xi)\right|^{2}\right.
\end{aligned}
$$




$$
\begin{gathered}
\left.\quad+|\xi|^{2|k|}\left|\hat{y}_{0}(\xi)\right|^{2}\right) d \xi \\
\leq C(1+t)^{-\frac{3}{2}-s-|k|-1}\left(\left\|\left(n_{0}, y_{0}\right)\right\|_{\dot{B}_{1, \infty}^{-s}\left(\mathbb{R}^{3}\right)}^{2}\right. \\
\left.\quad+\left\|\left(\partial_{x}^{|k|+1} n_{0}, \partial_{x}^{k} y_{0}\right)\right\|_{L^{2}\left(\mathbb{R}^{3}\right)}^{2}\right) \\
\leq C(1+t)^{-\frac{3}{2}-s-|k|-1}\left(\left\|\left(n_{0}, m_{0}\right)\right\|_{\dot{B}_{1, \infty}^{-s}\left(\mathbb{R}^{3}\right)}^{2}\right. \\
\left.\quad+\left\|\left(\partial_{x}^{|k|+1} n_{0}, \partial_{x}^{k} m_{0}\right)\right\|_{L^{2}\left(\mathbb{R}^{3}\right)}^{2}\right)
\end{gathered}
$$

and

$$
\begin{aligned}
&\left\|\partial_{x}^{k} y\right\|_{L^{2}\left(\mathbb{R}^{3}\right)}^{2}= \int_{\mathbb{R}^{3}}|\xi|^{2|k|} \mid \hat{g}_{21}(\xi, t) \hat{n}_{0}(\xi)+\left(\hat{g}_{22}(\xi, t)+\hat{g}_{2}(\xi, t)\right) \hat{y}_{0}(\xi) \\
& \leq C \int_{|\xi| \leq R} e^{-\beta|\xi|^{2} t}|\xi|^{2|k|}\left(\left|\hat{n}_{0}(\xi)\right|^{2}+\left|\hat{y}_{0}(\xi)\right|^{2}\right) d \xi \quad+C \int_{|\xi| \geq R} e^{-\beta|\xi|^{2} t}\left(|\xi|^{2(|k|+1)}\left|\hat{n}_{0}(\xi)\right|^{2}+|\xi|^{2|k|}\left|\hat{y}_{0}(\xi)\right|^{2}\right) d \xi \\
& \leq C(1+t)^{-\frac{3}{2}-s-|k|}\left(\left\|\left(n_{0}, y_{0}\right)\right\|_{\dot{B}_{1, \infty}^{-s}}^{2}\left(\mathbb{R}^{3}\right)\right. \\
&\left.\quad+\left\|\left(\partial_{x}^{|k|+1} n_{0}, \partial_{x}^{k} y_{0}\right)\right\|_{L^{2}\left(\mathbb{R}^{3}\right)}^{2}\right) \\
& \leq C(1+t)^{-\frac{3}{2}-s-|k|}\left(\left\|\left(n_{0}, m_{0}\right)\right\|_{\dot{B}_{1, \infty}^{-s}}^{2}\left(\mathbb{R}^{3}\right)\right. \\
&\left.\quad+\left\|\left(\partial_{x}^{|k|+1} n_{0}, \partial_{x}^{k} m_{0}\right)\right\|_{L^{2}\left(\mathbb{R}^{3}\right)}^{2}\right),
\end{aligned}
$$

for $0 \leqslant|k| \leqslant l$. By the relation between $m$ and $(y, \omega)$, we can easily get the estimate for $m$ :

$$
\left\|\partial_{x}^{k} m(t)\right\|_{L^{2}\left(\mathbb{R}^{3}\right)}^{2} \leq C(1+t)^{-\frac{3}{2}-s-|k|}\left(\left\|\left(n_{0}, m_{0}\right)\right\|_{\dot{B}_{1, \infty}^{-s}\left(\mathbb{R}^{3}\right)}^{2}+\left\|\left(\partial_{x}^{k+1} n_{0}, \partial_{x}^{k} m_{0}\right)\right\|_{L^{2}\left(\mathbb{R}^{3}\right)}^{2}\right) .
$$

The proof of Proposition 2.1 is completed.

It should be noted that the $L^{2}$-decay rates derived above are optimal. Indeed, we have:

Proposition 2.3. Assume that $n_{0} \in H^{l+1}\left(\mathbb{R}^{3}\right) \cap \dot{B}_{1, \infty}^{-s}\left(\mathbb{R}^{3}\right), m_{0} \in H^{l}\left(\mathbb{R}^{3}\right) \cap \dot{B}_{1, \infty}^{-s}\left(\mathbb{R}^{3}\right)$, $l \geq 3, s \in[0,1]$. Then, the solution $(n, m)$ to the IVP (2.1) given by Proposition 2.1 satisfies

$$
c_{1}(1+t)^{-\frac{3}{4}-\frac{s}{2}} \leq\|n(t)\|_{L^{2}\left(\mathbb{R}^{3}\right)}+\|m(t)\|_{L^{2}\left(\mathbb{R}^{3}\right)} \leq C(1+t)^{-\frac{3}{4}-\frac{s}{2}} .
$$

Proof. We only deal with the estimate of $\|m(t)\|_{L^{2}\left(\mathbb{R}^{3}\right)}$ for simplicity. From (2.18)-(2.20) we obtain

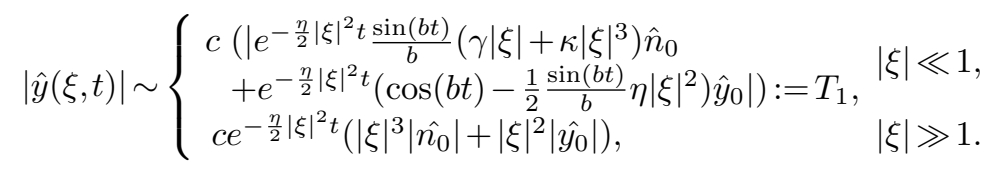

From Parseval's equality, we have

$$
\|y(t)\|_{L^{2}}^{2}=\|\hat{y}(t)\|_{L^{2}}^{2}=\int_{|\xi| \leq R}|\hat{y}(\xi, t)|^{2} d \xi+\int_{|\xi| \geq R}|\hat{y}(\xi, t)|^{2} d \xi
$$




$$
\begin{aligned}
& \geq c_{2} \int_{|\xi| \leq R}\left|T_{1}\right|^{2} d \xi-C e^{-\eta R^{2} t} \\
& \geq c_{3} \int_{|\xi| \leq R} e^{-\eta|\xi|^{2} t}|\xi|^{2 s}|\xi|^{4} t^{2} d \xi-C e^{-\eta R^{2} t} \\
& \geq c_{4}(1+t)^{-\frac{3}{2}-s} .
\end{aligned}
$$

By (2.4) and the pseudo-differential operators of degree zero, we obtain

$$
\begin{aligned}
\|m(t)\|_{L^{2}}^{2} & \geq c_{5}\left(\|y(t)\|_{L^{2}}^{2}-\|\omega(t)\|_{L^{2}}^{2}\right) \\
& \geq c_{5}(1+t)^{-\frac{3}{2}-s}-C(1+t)^{-\frac{3}{2}-s} \\
& \geq c_{1}(1+t)^{-\frac{3}{2}-s} .
\end{aligned}
$$

The time-decay rate of $n$ can be showed in a similar fashion.

\section{The proof of global well-posedness}

Let us reformulate the nonlinear system $(1.1)$ for $(\rho, m)$ near the equilibrium state $(\bar{\rho}, 0)=(1,0)$. Denote

$$
n=\rho-1, \quad m=m
$$

The IVP for $U:=(n, m)^{T}$ is

$$
\left\{\begin{array}{l}
\partial_{t} n+\nabla \cdot m=0 \\
\partial_{t} m-\mu \Delta m-\nu \nabla \operatorname{div} m+\gamma \nabla n-\kappa \nabla \Delta n=-\nabla \cdot H \\
\left.(n, m)\right|_{t=0}=\left(n_{0}, m_{0}\right)=\left(\rho_{0}(x)-1, m_{0}(x)\right), \quad x \in \mathbb{R}^{3}
\end{array}\right.
$$

where

$$
\begin{aligned}
H= & \frac{m \otimes m}{1+n}-\mu \nabla\left(\frac{n m}{1+n}\right)+\kappa \nabla n \otimes \nabla n+\left[-\nu \nabla \cdot\left(\frac{n m}{1+n}\right)\right. \\
& \left.+(P(1+n)-P(1)-\gamma n)-\kappa n \Delta n-\frac{1}{2} \kappa(\nabla n)^{2}\right] I_{3 \times 3} .
\end{aligned}
$$

As in Section 2, let $y:=\Lambda^{-1} \operatorname{div} m$ and $\omega:=\Lambda^{-1} \operatorname{curl} m$; we then have

$$
\left\{\begin{array}{l}
\partial_{t} n+\Lambda y=0 \\
\partial_{t} y-(\mu+\nu) \Delta y-\gamma \Lambda n-\kappa \Lambda^{3} n=-F \\
\partial_{t} \omega-\mu \Delta \omega=-G \\
n(x, 0)=n_{0}(x), y(x, 0)=\Lambda^{-1} \operatorname{div} m_{0}(x), \omega(x, 0)=\Lambda^{-1} \operatorname{curl} m_{0}(x), \quad x \in \mathbb{R}^{3},
\end{array}\right.
$$

where

$$
\begin{aligned}
& F=\Lambda^{-1} \operatorname{div} \nabla \cdot H, \\
& G=\Lambda^{-1} \operatorname{curl}\left(\nabla \cdot\left(\frac{m \otimes m}{1+n}-\mu \nabla\left(\frac{n m}{1+n}\right)+\kappa \nabla n \otimes \nabla n\right)\right) .
\end{aligned}
$$

If we denote

$$
\hat{B}_{1}(\xi, t)=\left(\hat{g_{11}}, \hat{g_{12}}\right), \hat{B}_{2}(\xi, t)=\left(\hat{g_{21}}+\hat{g_{1}}, \hat{g_{22}}+\hat{g_{2}}\right),
$$

then as in Section 2 we can represent the solution of system (3.2) as

$$
\omega(x, t)=\frac{1}{(4 \pi \mu t)^{\frac{3}{2}}} \int_{\mathbb{R}^{3}} e^{-\frac{|x-z|^{2}}{4 \mu t}} \omega_{0}(z) d z-\int_{0}^{t} \frac{1}{(4 \pi \mu(t-\tau))^{\frac{3}{2}}}\left(\int_{\mathbb{R}^{3}} e^{-\frac{|x-z|^{2}}{4 \mu(t-\tau)}} G(z, \tau) d z\right) d \tau,
$$




$$
\begin{aligned}
& n(x, t)=B_{1}(t) * V_{0}(x)+\int_{0}^{t} B_{1}(t-\tau) * Q(U)(\tau) d \tau, \\
& y(x, t)=B_{2}(t) * V_{0}(x)+\int_{0}^{t} B_{2}(t-\tau) * Q(U)(\tau) d \tau,
\end{aligned}
$$

with $V_{0}=\left(n_{0}, y_{0}\right)^{T}$ and $Q(U)=(0,-F)^{T}$.

It is easy to verify that

$$
\begin{array}{r}
n(x, t)=B_{1}(t) * V_{0}+\int_{0}^{t} B_{1}(t-\tau) * Q(U)(\tau) d \tau \\
m(x, t)=-\Lambda^{-1} \nabla y-\Lambda^{-1} \operatorname{div} \omega \\
=-\Lambda^{-1} \nabla\left\{B_{2}(t) * V_{0}(x)+\int_{0}^{t} B_{2}(t-\tau) * Q(U)(\tau) d \tau\right\} \\
-\Lambda^{-1} \operatorname{div}\left\{\frac{1}{(4 \pi \mu t)^{\frac{3}{2}}} \int_{\mathbb{R}^{3}} e^{-\frac{|x-z|^{2}}{4 \mu t}} \omega_{0}(z) d z\right. \\
\left.-\int_{0}^{t} \frac{1}{(4 \pi \mu(t-\tau))^{\frac{3}{2}}}\left(\int_{\mathbb{R}^{3}} e^{-\frac{|x-z|^{2}}{4 \mu(t-\tau)}} G(z, \tau) d z\right) d \tau\right\}
\end{array}
$$

is the solution of the IVP (3.1).

REMARK 3.1.

$$
\hat{\omega}(\xi, t)=e^{-\mu|\xi|^{2} t} \hat{\omega}_{0}(\xi, t)+\int_{0}^{t} e^{-\mu|\xi|^{2}(t-\tau)} \hat{G}(U)(\xi, \tau) d \tau .
$$

By Proposition 2.1, we have the following time decay rates for the linear parts:

$$
\begin{aligned}
&\left\|B_{1} * V_{0}(t)\right\|_{L^{2}\left(\mathbb{R}^{3}\right)} \leq C(1+t)^{-\frac{3}{4}-\frac{s}{2}}\left(\left\|V_{0}\right\|_{\dot{B}_{1, \infty}^{-s}\left(\mathbb{R}^{3}\right)}+\left\|V_{0}\right\|_{L^{2}\left(\mathbb{R}^{3}\right)}\right) \\
&\left\|\partial_{x}^{|\alpha|+1} B_{1} * V_{0}(t)\right\|_{L^{2}\left(\mathbb{R}^{3}\right) \leq} C(1+t)^{-\frac{3}{4}-\frac{s}{2}-\frac{|\alpha|+1}{2}}\left(\left\|V_{0}\right\|_{\dot{B}_{1, \infty}^{-s}\left(\mathbb{R}^{3}\right)}\right. \\
&+\left.\left\|\left(\partial_{x}^{|\alpha|+1} n_{0}, \partial_{x}^{\alpha} y_{0}\right)\right\|_{L^{2}\left(\mathbb{R}^{3}\right)}\right) \\
&\left\|\partial_{x}^{\alpha} B_{2} * V_{0}(t)\right\|_{L^{2}\left(\mathbb{R}^{3}\right)} \leq C(1+t)^{-\frac{3}{4}-\frac{s}{2}-\frac{|\alpha|}{2}}\left(\left\|V_{0}\right\|_{\dot{B}_{1, \infty}^{-s}\left(\mathbb{R}^{3}\right)}\right. \\
&\left.+\left\|\left(\partial_{x}^{|\alpha|+1} n_{0}, \partial_{x}^{\alpha} y_{0}\right)\right\|_{L^{2}\left(\mathbb{R}^{3}\right)}\right)
\end{aligned}
$$

for $|\alpha| \geq 0$. Applying arguments similar to those in the proof of Proposition 2.1, we can obtain that

$$
\begin{aligned}
& \left\|\partial_{x}^{\alpha} B_{1} * Q(U)(t)\right\|_{L^{2}\left(\mathbb{R}^{3}\right)} \\
\leq & C(1+t)^{-\frac{5}{4}-\frac{|\alpha|}{2}}\left(\|H(U)\|_{L^{\infty}\left(\mathbb{R}^{3}\right)}+\left\|\partial_{x}^{\alpha} H(U)\right\|_{L^{2}\left(\mathbb{R}^{3}\right)}\right), \\
& \left\|\partial_{x}^{\alpha} B_{1} * Q(U)(t)\right\|_{L^{2}\left(\mathbb{R}^{3}\right)} \\
\leq & C(1+t)^{-\frac{3}{4}-\frac{|\alpha|}{2}}\left(\|D H(U)\|_{L^{1}\left(\mathbb{R}^{3}\right)}+\left\|\partial_{x}^{\alpha} H(U)\right\|_{L^{2}\left(\mathbb{R}^{3}\right)}\right), \\
& \left\|\partial_{x}^{\alpha} B_{2} * Q(U)(t)\right\|_{L^{2}\left(\mathbb{R}^{3}\right)} \\
\leq & C(1+t)^{-\frac{5}{4}-\frac{|\alpha|}{2}}\left(\|\hat{H}(U)\|_{L^{\infty}\left(\mathbb{R}^{3}\right)}+\left\|\partial_{x}^{|\alpha|+1} H(U)\right\|_{L^{2}\left(\mathbb{R}^{3}\right)}\right),
\end{aligned}
$$




$$
\begin{aligned}
& \left\|\partial_{x}^{\alpha} B_{2} * Q(U)(t)\right\|_{L^{2}\left(\mathbb{R}^{3}\right)} \\
\leq & C(1+t)^{-\frac{3}{4}-\frac{|\alpha|}{2}}\left(\|D H(U)\|_{L^{1}\left(\mathbb{R}^{3}\right)}+\left\|\partial_{x}^{|\alpha|+1} H(U)\right\|_{L^{2}\left(\mathbb{R}^{3}\right)}\right),
\end{aligned}
$$

for $|\alpha| \geq 0$.

Global existence and $L^{2}$-decay rates. We are now ready to prove Theorem 1.2 and Theorem 1.3. First of all, the local existence and uniqueness of the solution can be established by following the methods in [20]. To prove global existence of a smooth solution with small initial data, we establish uniform a priori estimates of the solution.

Lemma 3.2. Under the assumption of Theorem 1.2, the solution $(n, m)$ to the IVP (3.1) satisfies for $l=3$ that

$$
\left\|D_{x}^{k}(n, m)(t)\right\|_{L^{2}\left(\mathbb{R}^{3}\right)} \leq C(1+t)^{-\frac{3}{4}-\frac{s}{2}-\frac{k}{2}}, k=0,1,\left\|D_{x}^{2} n(t)\right\|_{L^{2}\left(\mathbb{R}^{3}\right)} \leq C(1+t)^{-\frac{5}{4}-\frac{s}{2}} .
$$

Proof. Assume that $(n, m) \in H^{4}\left(\mathbb{R}^{3}\right) \times\left(H^{3}\left(\mathbb{R}^{3}\right)\right)^{3}$ corresponds to the strong solution to the IVP (3.1) for $t \in[0, T]$. Assume that the classical solution of the IVP (3.1) exists for $t \in[0, T]$, and denote

$$
\begin{aligned}
\Omega(t):=\sup _{0 \leq \tau \leq t, k=0,1}\left\{(1+\tau)^{\frac{3}{4}+\frac{s}{2}+\frac{k}{2}}\left\|D_{x}^{k}(n, m)(\tau)\right\|_{L^{2}\left(\mathbb{R}^{3}\right)}\right. \\
+(1+\tau)^{\frac{5}{4}+\frac{s}{2}}\left\|D_{x}^{2} n(\tau)\right\|_{L^{2}\left(\mathbb{R}^{3}\right)} \\
\left.+\left\|\left(D_{x}^{2} m, D_{x}^{3} m, D_{x}^{3} n, D_{x}^{4} n\right)\right\|_{L^{2}\left(\mathbb{R}^{3}\right)}\right\}
\end{aligned}
$$

We claim that it holds for any $t \in[0, T]$ that

$$
\Omega(t) \leq C \delta
$$

with $\delta$ defined in Theorem 1.2. The proof of the claim (3.16) consists of following three steps.

Step 1: The basic energy estimates From (3.4), (3.8), (3.11) and the a priori assumption (3.15), we have, after a complicated but straightforward computation, that

$$
\begin{aligned}
\left\|\left(n(t)-B_{1} * V_{0}(t)\right)\right\|_{L^{2}\left(\mathbb{R}^{3}\right)} \leq & \int_{0}^{t}\left\|B_{1}(t-\tau) * Q(U)(\tau)\right\|_{L^{2}\left(\mathbb{R}^{3}\right)} d \tau \\
\leq & C \int_{0}^{t}(1+t-\tau)^{-\frac{5}{4}}\left(\|\hat{H}(U)\|_{L^{\infty}\left(\mathbb{R}^{3}\right)}\right. \\
& \left.\left.+\|H(U)\|_{L^{2}\left(\mathbb{R}^{3}\right)}\right) d \tau\right) \\
\leq & C \int_{0}^{t}(1+t-\tau)^{-\frac{5}{4}}(1+\tau)^{-\frac{3}{2}-s}(\Omega(t))^{2} d \tau \\
\leq & C(1+t)^{-\frac{5}{4}}(\Omega(t))^{2},
\end{aligned}
$$

where we have made use of (3.15) and the Gagliardo-Nirenberg inequality

$$
\begin{gathered}
\|f\|_{L^{\infty}\left(\mathbb{R}^{3}\right)} \leq C\|D f\|_{L^{2}\left(\mathbb{R}^{3}\right)}^{\frac{1}{2}}\left\|D^{2} f\right\|_{L^{2}\left(\mathbb{R}^{3}\right)}^{\frac{1}{2}}, \\
\|f\|_{L^{4}\left(\mathbb{R}^{3}\right)} \leq C\|f\|_{L^{2}\left(\mathbb{R}^{3}\right)}^{\frac{1}{4}}\|D f\|_{L^{2}\left(\mathbb{R}^{3}\right)}^{\frac{3}{4}},
\end{gathered}
$$


to estimate the right-hand side term as

$$
\begin{aligned}
& \|\hat{H}(U)(t)\|_{L^{\infty}\left(\mathbb{R}^{3}\right)} \leq\|H(U)(t)\|_{L^{1}\left(\mathbb{R}^{3}\right)} \\
& \leq C\left\{\|m(t)\|_{L^{2}\left(\mathbb{R}^{3}\right)}^{2}+\|n(t)\|_{L^{2}\left(\mathbb{R}^{3}\right)}\|D m(t)\|_{L^{2}\left(\mathbb{R}^{3}\right)}\right. \\
& +\|D n(t)\|_{L^{2}\left(\mathbb{R}^{3}\right)}\|m(t)\|_{L^{2}\left(\mathbb{R}^{3}\right)}+\|n(t)\|_{L^{2}\left(\mathbb{R}^{3}\right)}^{2} \\
& \left.+\left\|D^{2} n(t)\right\|_{L^{2}\left(\mathbb{R}^{3}\right)}\|n(t)\|_{L^{2}\left(\mathbb{R}^{3}\right)}+\|D n(t)\|_{L^{2}\left(\mathbb{R}^{3}\right)}^{2}\right\} \\
& \leq C(1+t)^{-\frac{3}{2}-s}(\Omega(t))^{2} \text {, } \\
& \|H(U)(t)\|_{L^{2}\left(\mathbb{R}^{3}\right)} \leq C\left\{\|m(t)\|_{L^{4}\left(\mathbb{R}^{3}\right)}^{2}+\|D n(t)\|_{L^{4}\left(\mathbb{R}^{3}\right)}\|m(t)\|_{L^{4}\left(\mathbb{R}^{3}\right)}\right. \\
& +\|D m(t)\|_{L^{2}\left(\mathbb{R}^{3}\right)}\|n(t)\|_{L^{\infty}\left(\mathbb{R}^{3}\right)}+\|n(t)\|_{L^{4}\left(\mathbb{R}^{3}\right)}^{2} \\
& \left.+\left\|D^{2} n(t)\right\|_{L^{2}\left(\mathbb{R}^{3}\right)}\|n(t)\|_{L^{\infty}\left(\mathbb{R}^{3}\right)}+\|D n(t)\|_{L^{4}\left(\mathbb{R}^{3}\right)}^{2}\right\} \\
& \leq C(1+t)^{-\frac{9}{4}-s}(\Omega(t))^{2} \text {. }
\end{aligned}
$$

Thus, we have

$$
\begin{aligned}
\|n(t)\|_{L^{2}\left(\mathbb{R}^{3}\right)} & \leq\left\|B_{1} * V_{0}(t)\right\|_{L^{2}\left(\mathbb{R}^{3}\right)}+C(1+t)^{-\frac{5}{4}}(\Omega(t))^{2} \\
& \leq C(1+t)^{-\frac{3}{4}-\frac{s}{2}}\left[\delta+(\Omega(t))^{2}\right] .
\end{aligned}
$$

Similarly, we have

$$
\begin{aligned}
& \quad\|D H(U)(t)\|_{L^{2}\left(\mathbb{R}^{3}\right)} \\
& \leq C\left\{\|D m(t)\|_{L^{2}\left(\mathbb{R}^{3}\right)}\|m(t)\|_{L^{\infty}\left(\mathbb{R}^{3}\right)}+\|m(t)\|_{L^{\infty}\left(\mathbb{R}^{3}\right)}^{2}\|D n(t)\|_{L^{2}\left(\mathbb{R}^{3}\right)}\right. \\
& \quad+\left\|D^{2} n(t)\right\|_{L^{2}\left(\mathbb{R}^{3}\right)}\|m(t)\|_{L^{\infty}\left(\mathbb{R}^{3}\right)}+\left\|D^{2} n(t)\right\|_{L^{2}\left(\mathbb{R}^{3}\right)}\|D n(t)\|_{L^{\infty}\left(\mathbb{R}^{3}\right)} \\
& \quad+\|D m(t)\|_{L^{2}\left(\mathbb{R}^{3}\right)}\|D n(t)\|_{L^{\infty}\left(\mathbb{R}^{3}\right)}+\left\|D^{2} m(t)\right\|_{L^{2}\left(\mathbb{R}^{3}\right)}\|n(t)\|_{L^{\infty}\left(\mathbb{R}^{3}\right)} \\
& \left.\quad+\left\|D^{3} n(t)\right\|_{L^{2}\left(\mathbb{R}^{3}\right)}\|n(t)\|_{L^{\infty}\left(\mathbb{R}^{3}\right)}+\|D n(t)\|_{L^{2}\left(\mathbb{R}^{3}\right)}\|n(t)\|_{L^{\infty}\left(\mathbb{R}^{3}\right)}\right\} \\
& \leq C(1+t)^{-\frac{5}{4}-\frac{s}{2}}(\Omega(t))^{2},
\end{aligned}
$$

and

$$
\begin{aligned}
\|D n(t)\|_{L^{2}\left(\mathbb{R}^{3}\right)} \leq & \left\|D\left(B_{1} * V_{0}\right)(t)\right\|_{L^{2}\left(\mathbb{R}^{3}\right)}+\int_{0}^{t}\left\|D\left(B_{1} * Q(U)(\tau)\right)\right\|_{L^{2}\left(\mathbb{R}^{3}\right)} d \tau \\
\leq & C \delta(1+t)^{-\frac{3}{4}-\frac{s}{2}-\frac{1}{2}}+C \int_{0}^{t}(1+t-\tau)^{-\frac{5}{4}-\frac{1}{2}} \\
& \cdot\left(\|\hat{H}(U)(\tau)\|_{L^{\infty}\left(\mathbb{R}^{3}\right)}+\|D H(U)(\tau)\|_{L^{2}\left(\mathbb{R}^{3}\right)}\right) d \tau \\
& \leq C\left(\delta(1+t)^{-\frac{5}{4}-\frac{s}{2}}+\int_{0}^{t}(1+t-\tau)^{-\frac{7}{4}}(1+\tau)^{-\frac{5}{4}-\frac{s}{2}} \Omega^{2}(t) d \tau\right) \\
\leq & C(1+t)^{-\frac{5}{4}-\frac{s}{2}}\left[\delta+(\Omega(t))^{2}\right] .
\end{aligned}
$$

As for $D^{2} n$, it is easy to verify from the right-hand side of (3.4) that the nonlinear dominating terms consist of $D^{2}\left(\frac{m^{2}}{1+n}\right), D^{3}\left(\frac{n m}{1+n}\right)$ and $D^{2}(n \Delta n), D^{2}\left((\nabla n)^{2}\right)$, and can be estimated due to the following facts:

$$
\left\|D^{2}\left(\frac{m^{2}}{1+n}\right)\right\|_{L^{2}\left(\mathbb{R}^{3}\right)}+\left\|D^{3}\left(\frac{n m}{1+n}\right)\right\|_{L^{2}\left(\mathbb{R}^{3}\right)} \leq C(1+t)^{-\frac{5}{4}-\frac{s}{2}}(\Omega(t))^{2},
$$




$$
\left\|D^{2}(n \Delta n)\right\|_{L^{2}\left(\mathbb{R}^{3}\right)}+\left\|D^{2}\left((\nabla n)^{2}\right)\right\|_{L^{2}\left(\mathbb{R}^{3}\right)} \leq C(1+t)^{-\frac{5}{4}-\frac{s}{2}}(\Omega(t))^{2} .
$$

Thus, we can obtain after a straightforward computation that

$$
\left\|D^{2} H(U)(t)\right\|_{L^{2}\left(\mathbb{R}^{3}\right)} \leq C(1+t)^{-\frac{5}{4}-\frac{s}{2}}(\Omega(t))^{2},
$$

and

$$
\begin{aligned}
\left\|D^{2} n(t)\right\|_{L^{2}\left(\mathbb{R}^{3}\right)} \leq & \left\|D^{2}\left(B_{1} * V_{0}\right)(t)\right\|_{L^{2}\left(\mathbb{R}^{3}\right)}+\int_{0}^{t}\left\|D^{2}\left(B_{1}(t-\tau) * Q(U)(\tau)\right)\right\|_{L^{2}\left(\mathbb{R}^{3}\right)} d \tau \\
\leq & \quad C \delta(1+t)^{-\frac{7}{4}-\frac{s}{2}}+C \int_{0}^{t}(1+t-\tau)^{-\frac{9}{4}}\left(\|\hat{H}(U)(\tau)\|_{L^{\infty}\left(\mathbb{R}^{3}\right)}\right. \\
& \left.\quad+\left\|D^{2} H(U)(\tau)\right\|_{L^{2}\left(\mathbb{R}^{3}\right)}\right) d \tau \\
\leq & C \delta(1+t)^{-\frac{7}{4}-\frac{s}{2}}+C(1+t)^{-\frac{5}{4}-\frac{s}{2}}(\Omega(t))^{2} \\
\leq & \leq C(1+t)^{-\frac{5}{4}-\frac{s}{2}}\left[\delta+(\Omega(t))^{2}\right]
\end{aligned}
$$

As for $H$, we can obtain the estimates of $M$ :

$$
\begin{aligned}
& \|\hat{M}(U)(t)\|_{L^{\infty}\left(\mathbb{R}^{3}\right)} \leq C(1+t)^{-\frac{3}{2}-s}(\Omega(t))^{2}, \\
& \|D M(U)(t)\|_{L^{1}\left(\mathbb{R}^{3}\right)} \leq C(1+t)^{-2-s}(\Omega(t))^{2},
\end{aligned}
$$

where $M:=\frac{m \otimes m}{1+n}-\mu \nabla\left(\frac{n m}{1+n}\right)+\kappa \nabla n \otimes \nabla n$, so $G=\Lambda^{-1} \operatorname{curl}(\nabla \cdot M)$.

Now, from $s \in[0,1],(2.4),(3.3),(3.5),(3.10),(3.13),(3.21),(3.22),(3.25)$, the a priori assumption (3.15), and the Gagliardo-Nirenberg inequality, we can establish the time-decay rates for $m$ and its derivatives as follows:

$$
\begin{aligned}
& \|m(t)\|_{L^{2}\left(\mathbb{R}^{3}\right)} \\
\leq & C\left(\|y(t)\|_{L^{2}\left(\mathbb{R}^{3}\right)}+\|\omega(t)\|_{L^{2}\left(\mathbb{R}^{3}\right)}\right) \\
\leq & C\left(\left\|B_{2} * V_{0}(t)\right\|_{L^{2}\left(\mathbb{R}^{3}\right)}+\int_{0}^{t}\left\|B_{2}(t-\tau) * Q(U)(\tau)\right\|_{L^{2}\left(\mathbb{R}^{3}\right)} d \tau+\|\hat{\omega}(t)\|_{L^{2}\left(\mathbb{R}^{3}\right)}\right) \\
\leq & C(1+t)^{-\frac{3}{4}-\frac{s}{2}} \delta+C \int_{0}^{t}(1+t-\tau)^{-\frac{5}{4}}(1+\tau)^{-\frac{5}{4}-\frac{s}{2}}(\Omega(t))^{2} d \tau+C(1+t)^{-\frac{3}{4}-\frac{s}{2}} \delta \\
& \quad+C \int_{0}^{t}(1+t-\tau)^{-\frac{5}{4}}(1+\tau)^{-\frac{3}{2}-s}(\Omega(t))^{2} d \tau \\
\leq & C(1+t)^{-\frac{3}{4}-\frac{s}{2}}\left[\delta+(\Omega(t))^{2}\right] \\
& \|D m(t)\|_{L^{2}\left(\mathbb{R}^{3}\right)} \\
\leq & C\left(\|D y(t)\|_{L^{2}\left(\mathbb{R}^{3}\right)}+\|D \omega(t)\|_{L^{2}\left(\mathbb{R}^{3}\right)}\right) \\
\leq & C\left(\left\|D\left(B_{2} * V_{0}\right)(t)\right\|_{L^{2}\left(\mathbb{R}^{3}\right)}+\int_{0}^{t}\left\|D\left(B_{2}(t-\tau) * Q(U)(\tau)\right)\right\|_{L^{2}\left(\mathbb{R}^{3}\right)} d \tau\right. \\
& \left.+\|\xi \mid \hat{\omega}(t)\|_{L^{2}\left(\mathbb{R}^{3}\right)}\right) \\
\leq & C(1+t)^{-\frac{5}{4}-\frac{s}{2}} \delta+C \int_{0}^{t}(1+t-\tau)^{-\frac{7}{4}}(1+\tau)^{-\frac{5}{4}-\frac{s}{2}}(\Omega(t))^{2} d \tau+C(1+t)^{-\frac{5}{4}-\frac{s}{2} \delta} \\
& +C \int_{0}^{t / 2}(1+t-\tau)^{-\frac{7}{4}}(1+\tau)^{-\frac{3}{2}-s}(\Omega(t))^{2} d \tau
\end{aligned}
$$




$$
\begin{gathered}
+C \int_{t / 2}^{t}(1+t-\tau)^{-\frac{5}{4}}(1+\tau)^{-2-s}(\Omega(t))^{2} d \tau \\
\leq C(1+t)^{-\frac{5}{4}-\frac{s}{2}}\left[\delta+(\Omega(t))^{2}\right] .
\end{gathered}
$$

Step 2: The higher-order estimates. To enclose the a priori estimates and prove the claim (3.16), we need to derive the time-decay rates of $(n, m)$ with respect to the higher-order derivatives as in [19]. Indeed, using the energy methods, we can easily obtain

$$
\|n\|_{H^{4}\left(\mathbb{R}^{3}\right)}^{2}+\|m\|_{H^{3}\left(\mathbb{R}^{3}\right)}^{2}+\int_{0}^{t}\left(\|D n\|_{H^{4}\left(\mathbb{R}^{3}\right)}^{2}+\|D u\|_{H^{3}\left(\mathbb{R}^{3}\right)}^{2}\right) d \tau \leq C \delta^{2} .
$$

Step 3: Closure of the estimate (3.16). From (3.20), (3.22), (3.24), and (3.26)(3.28), we have

$$
\Omega(t) \leq C \delta+C(\Omega(t))^{2}, \quad t \in[0, T],
$$

which together with the smallness of $\delta>0$ leads to the estimate (3.16).

Similarly, we can obtain the following Lemmas, and omit the details.

Lemma 3.3. Under the assumption of Theorem 1.2, the solution $(n, m)$ to the IVP (3.1) satisfies for $l=4$ that

$$
\begin{array}{ll}
\left\|D_{x}^{k} n(t)\right\|_{L^{2}\left(\mathbb{R}^{3}\right)} \leq C(1+t)^{-\frac{3}{4}-\frac{s+k}{2}} \delta, & k=0,1, \\
\left\|D_{x}^{2} n(t)\right\|_{L^{2}\left(\mathbb{R}^{3}\right)}+\left\|D_{x}^{3} n(t)\right\|_{L^{2}\left(\mathbb{R}^{3}\right)} \leq C(1+t)^{-\frac{5}{4}-\frac{s}{2}} \delta, & \\
\left\|D_{x}^{k} m(t)\right\|_{L^{2}\left(\mathbb{R}^{3}\right)} \leq C(1+t)^{-\frac{3}{4}-\frac{s+k}{2}} \delta, & k=0,1, \\
\left\|D_{x}^{2} m(t)\right\|_{L^{2}\left(\mathbb{R}^{3}\right)} \leq C(1+t)^{-\frac{5}{4}-\frac{s}{2}} \delta, &
\end{array}
$$

where $C$ is a positive constant independent of time.

Lemma 3.4. Under the assumption of Theorem 1.2, the solution $(n, m)$ to the IVP (3.1) satisfies for $l=5$ that

$$
\begin{array}{ll}
\left\|D_{x}^{k} n(t)\right\|_{L^{2}\left(\mathbb{R}^{3}\right)} \leq C(1+t)^{-\frac{3}{4}-\frac{s+k}{2}} \delta, & k=0,1,2, \\
\left\|D_{x}^{3} n(t)\right\|_{L^{2}\left(\mathbb{R}^{3}\right)}+\left\|D_{x}^{4} n(t)\right\|_{L^{2}\left(\mathbb{R}^{3}\right)} \leq C(1+t)^{-\frac{5}{4}-\frac{s}{2}} \delta, & \\
\left\|D_{x}^{k} m(t)\right\|_{L^{2}\left(\mathbb{R}^{3}\right)} \leq C(1+t)^{-\frac{3}{4}-\frac{s+k}{2}} \delta, & k=0,1, \\
\left\|D_{x}^{2} m(t)\right\|_{L^{2}\left(\mathbb{R}^{3}\right)}+\left\|D_{x}^{3} m(t)\right\|_{L^{2}\left(\mathbb{R}^{3}\right)} \leq C(1+t)^{-\frac{5}{4}-\frac{s}{2}} \delta, &
\end{array}
$$

where $C$ is a positive constant independent of time.

REMARK 3.5.

$$
\begin{gathered}
\left\|D^{2} n(t)\right\|_{L^{2}\left(\mathbb{R}^{3}\right) \leq} \leq D^{2}\left(B_{1} * V_{0}\right)(t) \|_{L^{2}\left(\mathbb{R}^{3}\right)} \\
\quad+\int_{0}^{t}\left\|D^{2}\left(B_{1}(t-\tau) * Q(U)(\tau)\right)\right\|_{L^{2}\left(\mathbb{R}^{3}\right)} d \tau \\
\leq C \delta(1+t)^{-\frac{7}{4}-\frac{s}{2}}+C \int_{0}^{t / 2}(1+t-\tau)^{-\frac{9}{4}}\left(\|\hat{H}(U)(\tau)\|_{L^{\infty}\left(\mathbb{R}^{3}\right)}\right. \\
\left.\quad+\left\|D^{2} H(U)(\tau)\right\|_{L^{2}\left(\mathbb{R}^{3}\right)}\right) d \tau+C \int_{t / 2}^{t}(1+t-\tau)^{-\frac{7}{4}} \\
\quad \cdot\left(\|D H(U)(\tau)\|_{L^{1}\left(\mathbb{R}^{3}\right)}+\left\|D^{2} H(U)(\tau)\right\|_{L^{2}\left(\mathbb{R}^{3}\right)}\right) d \tau \\
\leq C \delta(1+t)^{-\frac{7}{4}-\frac{s}{2}}+C(1+t)^{-\frac{9}{4}}(\Omega(t))^{2}+C(1+t)^{-2-s}(\Omega(t))^{2} \\
\leq C(1+t)^{-\frac{7}{4}-\frac{s}{2}}\left[\delta+(\Omega(t))^{2}\right],
\end{gathered}
$$


for $l=5$, where

$$
\|D H\|_{L^{1}} \leq C(1+t)^{-2-s}(\Omega(t))^{2},\left\|D^{2} H\right\|_{L^{2}} \leq C(1+t)^{-\frac{5}{2}-s}(\Omega(t))^{2} .
$$

\section{Proof of Theorem 1.2 and Theorem 1.3.}

Proof. The global existence of smooth solutions to the IVP of the system (1.1) follows from the short-time existence of classical solution, the uniformly a priori estimates, and the continuity argument. The time-decay rate in Theorem 1.2 follows from the Lemma 3.2. The optimal time-decay rate of $\rho$ in Theorem 1.3 follows from the combination of the Proposition 2.3 and the uniform estimates (3.16). We also need to establish the lower bound decay rate for m. From (3.7) we have

$$
\begin{aligned}
\|m(t)\|_{L^{2}\left(\mathbb{R}^{3}\right)} \geq & c_{2}\|\hat{\omega}(t)\|_{L^{2}\left(\mathbb{R}^{3}\right)} \\
& \quad C\left(\left\|B_{2} * V_{0}(t)\right\|_{L^{2}\left(\mathbb{R}^{3}\right)}+\int_{0}^{t}\left\|B_{2}(t-\tau) * Q(U)(\tau)\right\|_{L^{2}\left(\mathbb{R}^{3}\right)} d \tau\right) \\
\geq & c_{3}(1+t)^{-\frac{3}{4}-\frac{s}{2}}-C \int_{0}^{t}(1+t-\tau)^{-\frac{5}{4}}(1+\tau)^{-\frac{3}{2}-s} d \tau \\
& \quad-c_{4}(1+t)^{-\frac{3}{4}-\frac{s}{2}}-C \int_{0}^{t}(1+t-\tau)^{-\frac{5}{4}}(1+\tau)^{-\frac{5}{4}-\frac{s}{2}} d \tau \\
\geq & c_{1}(1+t)^{-\frac{3}{4}-\frac{s}{2}} .
\end{aligned}
$$

Now the proof is completed.

\section{4. $L^{p}$-time-decay rates}

In this section, we investigate the $L^{p}$-time-decay rates of the solution to the system (3.1), with $p \in[2,+\infty]$. Making use of Lemma 3.4 and the Gagliardo-Nirenberg inequality, we can prove Theorem 1.4.

\section{The proof of Theorem 1.4.}

Proof. By Lemmas 3.3, 3.4 and the Gagliardo-Nirenberg inequality, (3.12), (3.14), we have

$$
\begin{gathered}
\|n(t)\|_{L^{\infty}\left(\mathbb{R}^{3}\right) \leq} \leq C\left\|B_{1} * V_{0}(t)\right\|_{L^{\infty}\left(\mathbb{R}^{3}\right)}+C \int_{0}^{t}\left\|B_{1}(t-\tau) * Q(U)(\tau)\right\|_{L^{\infty}\left(\mathbb{R}^{3}\right)} d \tau \\
\leq C(1+t)^{-\frac{3}{2}-\frac{s}{2}} \delta+C \int_{0}^{t}\left\|D\left(B_{1}(t-\tau) * Q(U)(\tau)\right)\right\|_{L^{2}\left(\mathbb{R}^{3}\right)}^{\frac{1}{2}} \\
\cdot\left\|D^{2}\left(B_{2}(t-\tau) * Q(U)(\tau)\right)\right\|_{L^{2}\left(\mathbb{R}^{3}\right)}^{\frac{1}{2}} d \tau \\
\leq C(1+t)^{-\frac{3}{2}-\frac{s}{2}} \delta+C \int_{0}^{t / 2}\left[(1+t-\tau)^{-\frac{7}{4}}\left(\|\hat{H}\|_{L^{\infty}}+\|D H\|_{L^{2}}\right)\right]^{\frac{1}{2}} \\
\cdot\left[(1+t-\tau)^{-\frac{9}{4}}\left(\|\hat{H}\|_{L^{\infty}}+\left\|D^{2} H\right\|_{L^{2}}\right)\right]^{\frac{1}{2}} d \tau \\
+C \int_{t / 2}^{t}\left[(1+t-\tau)^{-\frac{5}{4}}\left(\|D H\|_{L^{1}}+\|D H\|_{L^{2}}\right)\right]^{\frac{1}{2}} \\
\cdot\left[( 1 + t - \tau ) ^ { - \frac { 7 } { 4 } } \left(\|D H\|_{L^{1}}+\left\|D^{2} H\right\|_{\left.\left.L^{2}\right)\right]^{\frac{1}{2}} d \tau} d(1+t)^{-\frac{3}{8}-\frac{3}{4} s} \delta\right.\right. \\
\leq C(1+t)^{-\frac{3}{2}-\frac{s}{2}} \delta+C(1+t)^{-2} \delta+C(1+t)^{-\frac{3}{2}-\frac{s}{2}} \delta
\end{gathered}
$$

for $l \geq 4$, where

$$
\|D H\|_{L^{1}} \leq C(1+t)^{-2-s} \delta,\|D H\|_{L^{2}} \leq C(1+t)^{-\frac{5}{2}-s} \delta,\left\|D^{2} H\right\|_{L^{2}} \leq C(1+t)^{-\frac{5}{4}-\frac{s}{2}} \delta .
$$


and

$$
\begin{gathered}
\|y(t)\|_{L^{\infty}\left(\mathbb{R}^{3}\right)} \leq C\left\|B_{2} * V_{0}(t)\right\|_{L^{\infty}\left(\mathbb{R}^{3}\right)}+C \int_{0}^{t}\left\|B_{2}(t-\tau) * Q(U)(\tau)\right\|_{L^{\infty}\left(\mathbb{R}^{3}\right)} d \tau \\
\leq C(1+t)^{-\frac{3}{2}-\frac{s}{2}} \delta+C \int_{0}^{t}\left\|D\left(B_{2}(t-\tau) * Q(U)(\tau)\right)\right\|_{L^{2}\left(\mathbb{R}^{3}\right)}^{\frac{1}{2}} \\
\cdot\left\|D^{2}\left(B_{2}(t-\tau) * Q(U)(\tau)\right)\right\|_{L^{2}\left(\mathbb{R}^{3}\right)}^{\frac{1}{2}} d \tau \\
\leq C(1+t)^{-\frac{3}{2}-\frac{s}{2}} \delta+C \int_{0}^{t / 2}\left[(1+t-\tau)^{-\frac{7}{4}}\left(\|\hat{H}\|_{L^{\infty}}+\left\|D^{2} H\right\|_{L^{2}}\right)\right]^{\frac{1}{2}} \\
\cdot\left[(1+t-\tau)^{-\frac{9}{4}}\left(\|\hat{H}\|_{L^{\infty}}+\left\|D^{3} H\right\|_{L^{2}}\right)\right]^{\frac{1}{2}} d \tau \\
\quad+C \int_{t / 2}^{t}\left[(1+t-\tau)^{-\frac{5}{4}}\left(\|D H\|_{L^{1}}+\left\|D^{2} H\right\|_{L^{2}}\right)\right]^{\frac{1}{2}} \\
\cdot\left[(1+t-\tau)^{-\frac{7}{4}}\left(\|D H\|_{L^{1}}+\left\|D^{3} H\right\|_{L^{2}}\right)\right]^{\frac{1}{2}} d \tau \\
\leq C(1+t)^{-\frac{3}{2}-\frac{s}{2}} \delta+C(1+t)^{-2} \delta+C(1+t)^{-\frac{7}{4}-\frac{3}{4} s} \delta \\
\leq C(1+t)^{-\frac{3}{2}-\frac{s}{2}} \delta
\end{gathered}
$$

for $l \geq 5$, where

$$
\|D H\|_{L^{1}} \leq C(1+t)^{-2-s} \delta,\left\|D^{2} H\right\|_{L^{2}} \leq C(1+t)^{-\frac{5}{2}-s} \delta,\left\|D^{3} H\right\|_{L^{2}} \leq C(1+t)^{-\frac{3}{2}-\frac{s}{2}} \delta .
$$

So,

$$
\begin{aligned}
\|m(t)\|_{L^{\infty}\left(\mathbb{R}^{3}\right)} & \leq C\left(\|y(t)\|_{L^{\infty}\left(\mathbb{R}^{3}\right)}+\|\omega(t)\|_{L^{\infty}\left(\mathbb{R}^{3}\right)}\right) \\
& \leq C(1+t)^{-\frac{3}{2}-\frac{s}{2}} \delta
\end{aligned}
$$

where $C>0$ is a constant independent of time. From Theorem 1.2 and the interpolation, we can finish the proof of Theorem 1.4.

\section{REFERENCES}

[1] D. Bresch, B. Desjardins, and C.K. Lin, On some compressible fluid models: Korteweg, lubrication and shallow water systems, Commun. Part. Diff. Equ., 28, 843-868, 2003.

[2] R. Danchin and B. Desjardins, Existence of solutions for compressible fluid models of Korteweg type, Ann. Inst. Henri Poincare Anal. Non Linear, 18, 97-133, 2001.

[3] K. Deckelnick, Decay estimates for the compressible Navier-Stokes equations in unbounded domains, Math. Z., 209, 115-130, 1992.

[4] K. Deckelnick, $L^{2}$-decay for the compressible Navier-Stokes equations in unbounded domains, Commun. Part. Diff. Equ., 18, 1445-1476, 1993.

[5] J.E. Dunn and J. Serrin, On the thermomechanics of interstitial working, Arch. Rational Mech. Anal., 88, 95-133, 1985.

[6] Y. Guo and Y.J. Wang, Decay of dissipative equations and negative Sobolev spaces, Commun. Part. Diff. Equ., to appear.

[7] B. Haspot, Existence of global weak solution for compressible fluid models of Korteweg type, J. Math. Fluid Mech., 13, 223-249, 2011.

[8] H. Hattori and D. Li, Global solutions of a high-dimensional system for Korteweg materials, J. Math. Anal. Appl., 198, 84-97, 1996.

[9] H. Hattori and D. Li, Solutions for two-dimensional system for materials of Korteweg type, SIAM J. Math. Anal., 25, 85-98, 1994.

[10] S. Kawashima, Systems of a Hyperbolic-Parabolic Composite Type, with Applications to the Equations of Magnetohydrodynamics, Doctoral Thesis, Kyoto University, 1983.

[11] Y. Kagei and T. Kobayashi, Asymptotic behavior of solutions of the compressible Navier-Stokes equations on the half space, Arch. Ration. Mech. Anal. 177, 231-330, 2005.

[12] Y. Kagei and T. Kobayashi, On large time behavior of solutions to the compressible NavierStokes equations in the half space in $\mathbb{R}^{3}$, Arch. Ration. Mech. Anal., 165, 89-159, 2002. 
[13] T. Kobayashi, Some estimates of solutions for the equations of motion of compressible viscous fluid in an exterior domain in $\mathbb{R}^{3}$, J. Diff. Equ., 184, 587-619, 2002.

[14] T. Kobayashi and Y. Shibata, Decay estimates of solutions for the equations of motion of compressible viscous and heat-conductive gases in an exterior domain in $\mathbb{R}^{3}$, Commun. Math. Phys., 200, 621-659, 1999.

[15] D.J. Korteweg, Sur la forme que prennent les équations du mouvement des fluides si l'on tient compte des forces capillaires causées par des variations de densité considérables mais continues et sur la théorie de la capillarité dans l'hypothèse d'une variation continue de la densité, Archives Néerlandaises de Sciences Exactes et Naturelles, 1-24, 1901.

[16] M. Kotschote, Strong solutions for a compressible fluid model of Korteweg type, Ann. Inst. Henri Poincare Anal. Non Linear, 25, 679-696, 2008.

[17] H.L. Li, A. Matsumura, and G. Zhang, Optimal decay rate of the compressible Navier-StokesPoisson system in $\mathbb{R}^{3}$, Arch. Rat. Mech. Anal., 196, 681-713, 2010.

[18] H.L. Li and T. Zhang, Large time behavior of isentropic compressible Navier-Stokes system in $\mathbb{R}^{3}$, Math. Methods Appl. Sci., 34, 670-682, 2011.

[19] A. Matsumura and T. Nishida, The initial value problem for the equations of motion of compressible viscous and heat-conductive fluids, Proc. Japan Acad. Ser. A., 55, 337-342, 1979.

[20] A. Matsumura and T. Nishida, The initial value problems for the equations of motion of viscous and heat-conductive gases, J. Math. Kyoto Univ., 20, 67-104, 1980.

[21] G. Ponce, Global existence of small solution to a class of nonlinear evolution equations, Nonlin. Anal., 9, 339-418, 1985.

[22] Y. Shibata and K. Tanaka, Rate of convergence of non-stationary flow to the steady flow of compressible viscous fluid, Comput. Math. Appl., 53, 605-623, 2007.

[23] Z. Tan, Y. Wang, and X. Zhang, Large time behavior of solutions to the non-isentropic compressible Navier-Stokes-Poisson system in $\mathbb{R}^{3}$, Kinet. Relat. Mod. accepted.

[24] Y.J. Wang, Decay of the Navier-Stokes-Poisson equations, J. Diff. Equ., 253, 273-297, 2012.

[25] Y.J. Wang and Z. Tan, Global existence and optimal decay rate for the strong solutions in $H^{2}$ to the compressible Navier-Stokes equations, Appl. Math. Lett., 24, 1778-1784, 2011.

[26] Y.J. Wang and Z. Tan, Optimal decay rates for the compressible fluid models of Korteweg type, J. Math. Anal. Appl., 379, 256-271, 2011.

[27] G. Zhang, H.L. Li, and C. Zhu, Optimal decay rate for the non-isentropic compressible NaiverStokes-Poisson system in $\mathbb{R}^{3}$, J. Diff. Equ., 250, 866-891, 2011.

[28] Y. Zeng, $L^{1}$ asymptotic behavior of compressible isentropic viscous 1-D flow, Commun. Pure Appl. Math., 47, 1053-1082, 1994. 